\title{
Aprendizaje de los Conceptos Básicos de Realidad Aumentada por Medio del Juego Pokemon Go y sus Posibilidades como Herramienta de Mediación Educativa en Latinoamérica
}

Ronald S. Gutiérrez ${ }^{1}$, Erika T. Duque1, Ricardo L. Chaparro² y Néstor R. Rojas ${ }^{1}$

(1) Centro de Tecnologías para la Academia, Univ. de La Sabana, Campus Universitario, Chía, Colombia

(2) Universidad Manuela Beltrán, Campus Universitario Cajicá, Colombia.

(e-mail: ronaldgr@unisabana.edu.co; erika.duque@unisabana.edu.co; ricardo.chaparro@umb.edu.co; nestorromo@unisabana.edu.co)

Recibido May. 8, 2017; Aceptado Jul. 12, 2017; Versión final Sep. 12, 2017, Publicado Feb. 2018

\begin{abstract}
Resumen
El presente estudio tuvo como objetivo identificar el aprendizaje básico de la realidad aumentada que experimentaron los jugadores de Pokemon Go, así como las posibilidades que se observan en la Realidad Aumentada como herramienta de mediación educativa. Para ello, se desarrolló un instrumento aplicado a una población aleatoria de usuarios del juego. Se utilizó un enfoque cualitativo con diseño teoría fundamentada. Esto permitió observar que el juego ha generado un aprendizaje básico en los usuarios, el cual les permite comprender la base de su funcionamiento. Así mismo, se identificaron diferentes posibilidades de uso de la Realidad Aumentada como herramienta mediadora en procesos de aprendizaje.
\end{abstract}

Palabras clave: realidad aumentada; Pokemon GO; video juegos; aprendizaje básico

\section{Learning of the Basic Concepts of Augmented Reality through the Pokemon Go Game and its Possibilities as an Educational Mediation Tool in Latin America}

\begin{abstract}
The present study is aimed at identifying the basic learning of Augmented Reality experienced by Pokemon Go players as well as the possibilities observed in Augmented Reality as an educational mediation tool. Therefore, an instrument was designed and applied to a randomized population of users of this game. A qualitative approach with a grounded theory design was followed. This allowed observing that the game generated a basic learning in users, allowing them to understand the basis for its operation. Likewise, different possibilities of using Augmented Reality as a mediating tool in learning processes were identified.
\end{abstract}

Keywords: augmented reality; Pokemon GO; video games; basic learning 


\section{INTRODUCCIÓN}

Una de las tecnologías emergentes más prometedora en educación es la realidad aumentada (RA), considerada como una diversificación de entornos virtuales que incorpora objetos virtuales al mundo real (Azuma, 1997) y puede ser pensada como un intermedio entre un entorno virtual y la telepresencia completamente real (Milgram y Kishino, 1994). La RA permite el enriquecimiento de la realidad a través de la superposición de metadatos en formatos tales como texto, imagen, video y otros como coordenadas geográficas, que pueden ser visualizados a través de dispositivos de uso generalizado como celulares, tabletas y computadores (Johnson et al., 2016). Este enriquecimiento de la realidad a través de artefactos tecnológicos, se ha dado principalmente a través de los teléfonos móviles, donde por medio de la cámara, se puede ver en la pantalla el mundo real, al mismo tiempo que se agrega información relevante del mundo virtual por medio de una conexión a Internet, esto da origen al nombre de Realidad Aumentada (RA), término acuñado en 1992 por el ingeniero Tom Caudell (Lee, 2012). Existe una clasificación que hace referencia al espectro de la realidad versus virtualidad para clasificar los diferentes grados de "peso" que puede ofrecer esta tecnología en cuanto a la presencia de artefactos virtuales que pueden hacerla liviana o pesada de acuerdo al nivel de inmersión (Wu et al., 2013; Cheng y Tsai, 2013). Teniendo en cuenta este espectro, la realidad aumentada se clasificaría como liviana ya que requiere dispositivos de uso común y poco nivel de inmersión, lo cual hace que tenga una característica especialmente llamativa para efectos educativos por la relativa facilidad para acceder a ella desde los dispositivos móviles.

La RA se basa en metadatos multimediales, los cuales hacen referencia al enriquecimiento de la realidad aportando información pertinente para el usuario por medio de dispositivos de uso diario; esto puede ofrecer muchas oportunidades cognitivas que pueden ser aprovechadas ampliamente en el mundo educativo, como son por ejemplo la capacidad de visualizar conceptos abstractos y objetos tridimensionales. Aunque generalmente se realiza el vínculo entre imágenes o figuras tridimensionales, también puede utilizarse sonidos, generar aromas o sensaciones táctiles (Fabregat, 2012). En este sentido existen muchos referentes de uso educativo: Cuendet et al. (2013) hacen referencia al uso de esta tecnología para visualización de figuras en 3D para el entrenamiento en carpintería, mostrando la motivación y la comprensión espacial sobre todo para la elaboración de planos técnicos. Andújar et al. (2011) describen la potencialidad de la realidad aumentada en la implementación de laboratorios remotos, sin embargo una de las mayores potencialidades tiene que ver con las estrategias de juegos. El Informe Horizon de 2016 publicado por Johnson et al. (2016), muestra que la realidad aumentada se vislumbra como una aplicación que se incorporará en las aulas al año 2020. Este hecho presenta un reto para los docentes, quienes deberán estar preparados para romper exitosamente los esquemas tradicionales, (Marín, 2016) gracias a que esta tecnología multimedial permite la exploración de lenguajes expresivos amplios, que podrían ser aprovechados para efectos pedagógicos, teniendo en cuenta que los estudiantes son consumidores y al mismo tiempo, productores de este tipo de recursos (Área y Guarro, 2012).

Billinghurst y Duenser (2012) han mostrado la potencialidad de los libros interactivos utilizando realidad aumentada para fomentar las competencias de comprensión de lectura en los niños de escuelas a través de esta tecnología. En cuanto a los museos interactivos, Barry et al. (2012), hacen referencia a la potencialidad educativa que ofrecen dichos museos, entre los que se destaca la experiencia en el Museo de Londres donde las imágenes interactivas ofrecen capacidades de recordación, tal como lo indica Torres (2011) al resaltar las aplicaciones en los museos y el valor de la experiencia de la interacción entre los visitantes. Algunos autores como Estebanell et al. (2012) afirman que esta tecnología permite disfrutar de experiencias globales, como la aplicación del juego Pokémon Go para dispositivos móviles, creado para capturar personajes por todo el mundo llamados Pokemones y que para permitirlo utiliza el GPS del celular con un sistema de geolocalización cambiando la forma de interactuar con los videojuegos y generalizando la realidad aumentada a nivel global (Sanchez, 2016). Aún más, con la aparición del juego en el año 2016 se considera una forma diversa de aprendizaje que incorpora el concepto de realidad aumentada a la captura de personajes animados con ayuda de dispositivos móviles por todo el mundo, transcendiendo entornos políticos, sociales y educativos.

En este sentido, se reconocen las bondades del mundo Pokémon, desarrollado por la compañía Game Freak, quien lanzó sus primeros productos en el año 1996 y ha incluido a través de los años videojuegos, juegos de cartas coleccionables, dibujos animados, películas, juguetes, entre otros, de tal manera que permiten: despertar las habilidades en matemáticas básicas, fomentar el pensamiento estratégico, incorporar las habilidades motoras, enseñar el respeto por los otros contrincantes y fomentar la lectura (Guía de Pokémon para padres / tutores, 2016). Como afirma Díaz (2016) "fortalece la atención, concentración, memoria inmediata (largo plazo) en sus formas visuales y auditivas, así como del razonamiento ... activación de procesos cognitivos de aprendizaje" (p. 2). En consecuencia, la realidad aumentada promueve la curiosidad y motivación en los estudiantes (Wu et al., 2013) lo cual ofrece dos aspectos para ser analizados desde el punto de vista educativo: una ventaja en la captación de la atención de los estudiantes o una forma de distracción (Cheng y Tsai, 2013). Así mismo, Pokémon es una posibilidad de conocer la realidad aumentada 
a través de juegos que motiven la interacción. Existen reflexiones importantes en las diferentes áreas del conocimiento que ayudan al docente a utilizar este juego como una herramienta educativa, por ejemplo, en el caso del área de humanidades se pueden crear historietas, comics, narrativas digitales y textos descriptivos con solo capturar pantallas en los sitios donde se encuentren los pokemones. Otro ejemplo, es obtener datos de las capturas de coordenadas y localización de sitios que permitirían diseñar encuestas con otros participantes y graficar datos, aportando estrategias innovadoras en cuanto al aprendizaje de las matemáticas y estadística aplicadas (Universia, 2016). También, es posible incorporar datos geográficos donde se puedan extraer las características de clima, espacio, coordenadas, hábitat, entre otros (Universia, 2016) y que podrían contribuir al aprendizaje de geografía.

De esta forma, la utilización de Pokémon Go en el campo educativo, de acuerdo a Araya (2017) sirve para capturar la atención de los estudiantes, ya que es una buena manera para mantenerlos interesados e involucrados con los temas, por lo tanto, se puede considerar que las aplicaciones de Pokémon GO se incorporen en las escuelas, como lo hizo la profesora de biología de la escuela de educación técnica Marisol Biso, quién adaptó la aplicación, para enseñar contenidos de genética. Lo más importante es que estas tecnologías, puedan incorporarse desde el aula a las necesidades de aprendizaje de los estudiantes y para ello el profesor debe emplear su creatividad y los recursos tecnológicos que tiene a la mano con el fin de aprovechar los usos educativos que ofrecen estas tecnologías y que son tema de investigación actual (Araya, 2017).

\section{METODOLOGÍA}

El presente estudio está enfocado en comprender la influencia que ha tenido el juego Pokémon Go en el aprendizaje de los conceptos básicos de la RA y las posibilidades que ésta tiene como mediación tecnológica en procesos educativos. Se planteó una investigación con enfoque cualitativo utilizando para ello un cuestionario distribuido por Internet en diferentes grupos de Facebook latinoamericanos, dedicados a los jugadores de la aplicación. Se recolectaron 45 registros de diferentes personas con perfiles heterogéneos.

\section{Población}

La población fue aleatoria a conveniencia, debido a la forma de convocatoria que se realizó. El criterio de selección fue a través de un incentivo: los jugadores que participaran en el cuestionario recibirían una mini figura de pokémon. Los datos que caracterizan la población se muestran en la tablas 1 y 2 . De la tabla 1 se destaca una mayoría del sexo masculino correspondiente al $82 \%$, con un nivel educativo mayoritario universitario de $58 \%$. De igual manera se observa que un $67 \%$ del grupo aún jugaba en el momento del estudio, mientras que un 33\% ya habían dejado de utilizar la aplicación. En cuanto al tipo de respuesta "si, pero menos que al comienzo", se puede observar que el $36 \%$ lo siguen jugando con una frecuencia similar a la del comienzo, mientras que $33 \%$ lo jugaron mucho al comienzo pero han reducido su frecuencia de juego. De la tabla 2 se destaca que la media de edad es de 25.1 años, así como el nivel promedio (nivel logrado dentro del juego) de los jugadores es 17, con un promedio de 150 días de haber comenzado a jugar (los días jugando se han tomado desde el día que el jugador comenzó a utilizar la aplicación hasta la fecha 6 de enero de 2017, cuando se realizó el cierre de la captura de datos del estudio).

Tabla 1: Género y nivel educativo de la población

\begin{tabular}{lll}
\hline \multicolumn{1}{c}{ Variable } & Frecuencia & Porcentaje \\
\hline Sexo & & \\
Mujeres & 8 & $18 \%$ \\
Hombres & 37 & $82 \%$ \\
Nivel educativo & & \\
Colegio & 5 & $11 \%$ \\
Universitario & 26 & $58 \%$ \\
Postgrado & 8 & $18 \%$ \\
NS/NR & 6 & $13 \%$ \\
Juega Actualmente & & \\
Si & 16 & $36 \%$ \\
Sí, pero menos que al comienzo & 14 & $31 \%$ \\
No & 15 & $33 \%$ \\
\hline
\end{tabular}


Tabla 2: Edad y condiciones de juego

\begin{tabular}{lllll}
\hline Variable & Menor & Mayor & Media & Desv Est \\
\hline Edad (años) & 10 & 47 & 25,1 & 8 \\
Nivel de Juego & 1 & 29 & 17 & 8,5 \\
Días Jugando & 19 & 244 & 150 & 50 \\
\hline
\end{tabular}

Método de evaluación

Para la investigación, se diseñó un cuestionario de tipo cualitativo (tabla 3), aplicado en la red social Facebook. Dicho instrumento fue validado por un experto y posteriormente con un grupo de 5 personas a modo de pilotaje. El cuestionario tiene algunas preguntas de tipo demográfico, las preguntas 4 hasta la número 9 son abiertas y la pregunta 10 es categorial. El cuestionario fue desarrollado de manera online con la aplicación del software libre GoogleForms sobre la plataforma Facebook.

Tabla 3: Instrumento cuestionario

\begin{tabular}{cl}
\hline Número & \multicolumn{1}{c}{ Pregunta } \\
\hline 4 & ¿Cuál es la razón por la que ya no juegas Pokémon GO o por la cual juegas menos ahora? \\
5 & ¿Qué es la Realidad Aumentada (RA)? \\
7 & ¿Cómo funciona la RA? \\
8 & ¿Qué elementos tecnológicos se requieren para que la RA funcione? \\
9 & ¿Qué usos sociales se le pueden dar a la RA? \\
10 & ¿Cómo define su aprendizaje sobre RA mientras jugaba PG?
\end{tabular}

El análisis de datos se desarrolló por medio la metodología de investigación denominada teoría fundamentada (Strauss y Corbin, 2002), donde los datos se obtienen de descripciones y ordenamientos conceptuales de respuestas. Estas fueron analizadas y agrupadas de forma sistemática, para lograr la posibilidad de brindar interpretaciones hasta llegar a obtener inferencias que pudieran respaldar la validez y confiabilidad, por medio de una óptima teorización en la investigación.

De igual forma se desarrolló dentro de la metodología un orden sistemático, del cual se describieron las categorías a priori y las categorías emergentes. Lo anterior, hace parte de la codificación abierta. Esta codificación fue un procedimiento analítico, donde se logró indagar los conceptos descritos en el cuestionario, que permitieron descubrir propiedades y dimensiones según las respuestas. Luego, por medio del software Atlas TI se desarrolló la codificación axial, donde se relacionaron categorías y subcategorías y se analizó el número de veces que aparecen los nuevos códigos, que surgieron de las respuestas del cuestionario. Lo anterior, encontrando nuevas categorías y subcategorías que apoyaron igualmente la codificación emergente y de esta forma se culminó con la codificación selectiva por medio del surgimiento de inferencias que aportaron confiabilidad en el desarrollo de las conclusiones del artículo.

Para describir las categorías a priori, se analizaron los conceptos recurrentes que se obtuvieron desde la problemática y antecedentes descritos en las respuestas del cuestionario (Strauss y Corbin, 2002). Luego, las categorías emergentes se obtuvieron por la similitud y frecuencia que se alcanzaron de las respuestas 4 a 9 del cuestionario (Tabla 4).

Tabla 4: Categorías de análisis

\begin{tabular}{l}
\hline Categorías \\
\hline Categorías Emergentes: \\
Insatisfacción \\
Acceso a nuevas tendencias de información digital \\
Posicionamiento espacial por medio de imágenes en tiempo real \\
Simuladores \\
Categorías A priori: \\
Intercambio de información para un mayor conocimiento \\
Generación de experiencias en comunidad
\end{tabular}




\section{Definición de las categorías A priori y Emergentes, según el contexto de la investigación}

Insatisfacción: La tecnología digital contiene diversidad de usuarios insatisfechos. Lo anterior, a causa de que no se logran obtener optimas experiencias en los juegos interactivos, que no cumplen con las expectativas, ya sea por la monotonía o por dinámicas poco colaborativas entre usuarios (De Acevedo, 2014).

Acceso a nuevas tendencias de información digital: La expansión y acceso a las tecnologías digitales, mejora las opciones que existen actualmente para obtener información por medio de la inclusión e innovación. Igualmente, las nuevas tendencias digitales, brindan oportunidades que antes estaban fuera del alcance en sectores desfavorecidos. Es de mencionar, que la web permite incrementar la productividad en la educación, el entretenimiento y la economía. (Banco Mundial, 2016)

Posicionamiento espacial por medio de imágenes en tiempo real: Es la ubicación de un objeto en el espacio representado en mapas de posición y reconocimiento en tiempo real. Lo anterior, desde la activación de una geo localización para tener una posición exacta, Esta tecnología se ha convertido en una herramienta de comunicación a través de la nube (Beltrán, 2016).

Simuladores: Es una comparación que se asemeja a la realidad y en la actualidad tiene mayor acercamiento hacia el ser humano como recurso didáctico de información y aprendizaje. Desde el aspecto digital, los simuladores son configuraciones de software y hardware que, mediante algoritmos de cálculo, evidencian un comportamiento determinado por medio de procesos que se asemejan a los aspectos físicos (Dockterman, 1997)

Intercambio de información para un mayor conocimiento: conocimiento e información son componentes primordiales en el desarrollo e intercambio de información; "el término informacional indica el atributo de una forma específica de organización social en la que la generación, el procesamiento y la transmisión de información se convierten en las fuentes fundamentales de la productividad y el poder". (Castells 1999, p.47.)

Generación de experiencias en comunidad: el concepto comunidad de práctica de experiencias fue mencionado por Wenger (2001), donde asocia el desarrollo de la práctica a un contexto, con el objetivo de promover el propósito de diferenciar del concepto más extenso de cultura, hasta interpretar como es un tipo objetivo de comunidad. El autor indica que las dimensiones de la medición en la práctica que pueden ayudar a una sociedad pueden ser, el compromiso reciproco, la empresa conjunta y el catalogo compartido. Lo anterior, con la finalidad de constituir un entorno de aprendizaje en una sociedad que ha de favorecer el deber y la adopción de sus segmentos por medio de una infraestructura para la participación que incorpore múltiples medios para promover la competencia y la mutualidad (Wenger, 2001).

\section{RESULTADOS}

A continuación se describe el análisis de datos y los resultados de las inferencias, producto del análisis cualitativo de las preguntas 4 a 9 tomando como base las categorías emergentes y a priori. El procedimiento sistemático de la metodología fue implementado con el software Atlas $\mathrm{TI}$, para el procesamiento de los datos. También se describe el análisis de los resultados de la pregunta 10, la cual es de tipo categorial y que fue procesada en el software SPSS.

\section{Categoría Insatisfacción}

Esta categoría está asociada con las subcategorías monotonía y competitividad.

Cuestionario, pregunta aplicada en la red social Facebook. No 4. ¿Cuál es la razón por la que ya no juegas Pokémon GO o por la cual juegas menos ahora? A partir del análisis cualitativo se pudo identificar la relación como se muestra en la tabla 5.

Tabla 5: Categorías para pregunta 4

\begin{tabular}{lll}
\hline Categoría & Subcategoría & \multicolumn{1}{c}{ Inferencias } \\
\hline Monotonía. & $\begin{array}{l}\text { La misma rutina en } \\
\text { espacios de tiempo. }\end{array}$ \\
Insatisfacción. & Bajo interés en el juego. \\
& Competitividad. & $\begin{array}{l}\text { Implementar más } \\
\text { dinámicas de manera } \\
\text { colaborativa. }\end{array}$ \\
\hline
\end{tabular}


Inferencias respecto a las categorías y subcategorías: se evidencia en las respuestas de los usuarios respecto a la pregunta 4 y para complementar las categorías y subcategorías de la tabla 5 , una dinámica de juego que se convierte en rutina por parte del grupo objetivo, el cual repercute en una constante respecto a la interfaz en los espacios de tiempo que contiene la aplicación. Lo anterior influye en un bajo interés en el juego. Igualmente se sugiere implantar mayores dinámicas de manera colaborativa.

Se evidencia en la siguiente segmentación, una muestra de las respuestas que justifica la inferencia:

Persona 1: "Me está aburriendo, solo se encuentran los mismos Pokemón. Crea tecno dependencia"

Persona 2: "Me desmotiva no pelear con mis amigos en tiempo real y las peleas en gimnasios son "al azar". Es mejor el sistema por turnos de Gameboy"

Persona 3: "Falta implementar más elementos, sobretodo asuntos competitivos. Monotonía en el juego"

Categoría Acceso a nuevas tendencias de información digital

Esta categoría está asociada con las subcategorías: combinación de herramientas virtuales con el mundo real, estrategias tecnológicas didácticas para obtener información.

Cuestionario, pregunta aplicada en la red social Facebook. No5. ¿Qué es la Realidad Aumentada (RA)?. A partir del análisis cualitativo se pudo identificar la siguiente relación

Tabla 6: Categorías para pregunta 5

\begin{tabular}{cll}
\hline \multicolumn{1}{c}{ Categoría } & \multicolumn{1}{c}{ Subcategoría } & \multicolumn{1}{c}{ Inferencias } \\
\hline $\begin{array}{l}\text { Acceso a nuevas tendencias de } \\
\text { información digital. }\end{array}$ & $\begin{array}{l}\text { Combinación de herramientas } \\
\text { virtuales con el mundo real. }\end{array}$ & $\begin{array}{l}\text { Análisis de objetos animados en un } \\
\text { ambiente real por medio de un dispositivo. }\end{array}$
\end{tabular}

Estrategias tecnológicas para

Información en base a sensaciones que obtener información. alteraron los sentidos de la realidad.

Inferencias respecto a las categorías y subcategorías: se evidencia en las respuestas que proporcionan los usuarios respecto a la pregunta 5 y para complementar las categorías y subcategorías de la tabla 6 , que la RA es un análisis de objetos animados en un ambiente real, por medio de un dispositivo móvil y que la información se describe en base a nuevas sensaciones que alteran los sentidos de la realidad.

Se evidencia en la siguiente segmentación, una muestra de las respuestas que justifica la inferencia:

Persona 4: "Combinación imágenes reales con virtuales"

Persona 5: "Aprovechamiento de herramientas digitales sobre elementos tangibles que permiten crear la sensación de estar en nuevos escenarios"

Persona 6: "La realidad aumentada consiste en combinar el mundo real con el virtual mediante un proceso informático, enriqueciendo la experiencia visual y mejorando la calidad de comunicación"

Categoría Posicionamiento espacial por medio de imágenes en tiempo real

Esta categoría está asociada con la subcategoría: software de reconocimiento que puede ser identificado por códigos tipo QR.

Cuestionario, pregunta aplicada en la red social Facebook. No 6. ¿Cómo funciona la RA?. A partir del análisis Cualitativo se pudo identificar la siguiente relación:

Tabla 7: Tabla de categorías para pregunta 6

\begin{tabular}{lll}
\hline \multicolumn{1}{c}{ Categoría } & Subcategoría & \multicolumn{1}{c}{ Inferencia } \\
\hline $\begin{array}{l}\text { Posicionamiento espacial por medio } \\
\text { de imágenes en tiempo real. }\end{array}$ & $\begin{array}{l}\text { Software de reconocimiento que } \\
\text { puede ser identificación por códigos. }\end{array}$ & $\begin{array}{l}\text { Uso de la web y en ocasiones } \\
\text { aplicaciones que necesitan } \\
\text { geolocalización. }\end{array}$ \\
\hline
\end{tabular}


Inferencias respecto a las categorías y subcategorías: se evidencia en las respuestas que proporcionan los usuarios respecto a la pregunta 6 y para complementar las categorías y subcategorías de la tabla 7, el adecuado conocimiento respecto a la implementación de aplicativos móviles con ayuda de la web y el uso de características detalladas de geo-localización, como es el juego denominado Pokémon GO.

Se evidencia en la siguiente segmentación, una muestra de las respuestas que justifica la inferencia:

Persona 7: "Un lector nutre de información el programa y genera una respuesta para el usuario en ese mismo dispositivo. Por medio de software de reconocimiento de códigos, puede ser geo-localizados"

Persona 8: "Se reproduce un video en una pantalla de un dispositivo y se combina con el video del mundo real con la cámara se capta el mundo real y al visualizar se incluye la capa virtual"

\section{Categoría Simuladores}

Esta categoría está asociada con la subcategoría, función por medio de dispositivos móviles.

Cuestionario, pregunta aplicada en la red social Facebook. No7. ¿Qué elementos tecnológicos se requieren para que la RA funcione? A partir del análisis Cualitativo se pudo identificar la siguiente relación.

Tabla 8: categorías para pregunta 7

\begin{tabular}{lll}
\hline Categoría & \multicolumn{1}{c}{ Subcategoría } & \multicolumn{1}{c}{ Inferencias } \\
\hline \multirow{2}{*}{ Simuladores } & Función por medio de dispositivos móviles & Smart phone, tabletas, computadores \\
& & Apps. \\
\hline
\end{tabular}

Inferencias respecto a las categorías y subcategorías: Se evidencia en las respuestas que proporcionan los usuarios respecto a la pregunta 7 y para complementar las categorías y subcategorías de la tabla 8 , el uso de una adecuada comunicación entre el emisor y el receptor por medio de dispositivos como, Smarthphone, Tablets, Computadores. Lo anterior incorporando las APP's como componentes didácticos en la emisión de información hacia la población.

Se evidencia en la siguiente segmentación, una muestra de las respuestas que justifica la inferencia:

Persona 9: "La cámara del dispositivo"

Persona 10: "Dispositivo inteligente y software que facilite la interacción con conexión a internet"

Categoría Intercambio de información para un mayor conocimiento

Esta categoría está asociada con las subcategorías, educación, entretenimiento.

Cuestionario, pregunta aplicada en la red social Facebook. No8. ¿Qué usos sociales se le pueden dar a la RA?. A partir del análisis Cualitativo se pudieron identificar la siguientes relaciones.

Tabla 9: Categorías para pregunta 8.

\begin{tabular}{lll}
\hline \multicolumn{1}{c}{ Categoría } & Subcategoría & \multicolumn{1}{c}{ Inferencias } \\
\hline $\begin{array}{lll}\text { Intercambio de información para un mayor } \\
\text { conocimiento. }\end{array}$ & Educación. & $\begin{array}{l}\text { Acceso a información en tiempo real sin límites de } \\
\text { tiempo y espacio. }\end{array}$ \\
\hline
\end{tabular}

Inferencias respecto a las categorías y subcategorías: Se evidencia en las respuestas que proporcionan los usuarios respecto a la pregunta 8 y para complementar las categorías y subcategorías de la tabla 9 , un uso social colectivo por medio de acceso a información en tiempo real sin límites de tiempo y espacio en tiempo real. Lo anterior fomentando el uso de las redes sociales para la participación en comunidad.

Se evidencia en la siguiente segmentación, una muestra de las respuestas que justifica la inferencia:

Persona 11: "Poder aplicarlo todas las redes sociales, pues se pueden tener los espacios en el tiempo real y tener más cerca a las personas de manera sincrónica." 
Persona 12: "Las posibilidades sociales son infinitas para las mentes creativas, gracias a la constante expansión de la tecnología y la web."

\section{Categoría Generación de experiencias en comunidad}

Esta categoría está asociada con la subcategorías, video juegos significativos, video juego de aprendizaje.

Cuestionario, pregunta aplicada en la red social Facebook. No9. ¿Qué video juego crearías con RA?. A partir del análisis Cualitativo se pudieron identificar la siguiente relación

Tabla 10: Categorías para pregunta 9.

\begin{tabular}{lll}
\hline \multicolumn{1}{c}{ Categoría } & \multicolumn{1}{c}{ Subcategoría } & \multicolumn{1}{c}{ Inferencias } \\
\hline $\begin{array}{l}\text { Generación de experiencias en } \\
\text { comunidad. }\end{array}$ & $\begin{array}{l}\text { Videojuegos de } \\
\text { aprendizaje. }\end{array}$ & Aprendizaje con mayor realidad sensorial. \\
& $\begin{array}{l}\text { Aplicación de nuevos retos en los videojuegos con RA } \\
\text { significativos. }\end{array}$ & $\begin{array}{l}\text { para mayor interactividad. } \\
\text { Mejoras en el juego de Pokémon Go implementado en } \\
\end{array}$ \\
& RA. \\
\hline
\end{tabular}

Inferencias respecto a las categorías y subcategorías: Se evidencia en las respuestas que proporcionan los usuarios respecto a la pregunta 9 y para complementar las categorías y subcategorías de la tabla 10, la aplicación de nuevos retos en los videojuegos con RA para mayor interactividad. Igualmente describen mejoras en el juego de Pokémon Go. Lo anterior incorporando un aprendizaje con mayor realidad sensorial por medio de la interactividad en la población objetivo del estudio.

Se evidencia en la siguiente segmentación, una muestra de las respuestas que justifica la inferencia:

Persona 13: "Juegos académicos, para hacer más real los aprendizajes"

Persona 14: "Juegos de estrategia que permitan el desarrollo de habilidades específicas en una temática determinada a partir de un recorrido por divertidos lugares de una institución."

Persona 15: "Un juego que permita el desarrollo cognitivo y sensorial de las personas con el fin que puedan desarrollar conocimientos implementando tecnologías"

\section{Aspectos cuantitativos}

Respecto a los resultados de la pregunta de apoyo cuantitativo en la tabla 11 se pueden observar los resultados obtenidos en la pregunta 10, acerca de la percepción que tienen los participantes frente a lo que aprendieron sobre la RA durante su experiencia de juego. Se puede resaltar que un $85 \%$ de la población se encuentra en los rangos de Muy satisfactorio, Satisfactorio y Ni satisfactorio Ni deficiente.

Tabla 11: Resultados de la pregunta 10 del cuestionario.

\begin{tabular}{lll}
$\begin{array}{l}\text { ¿Cómo define su aprendizaje sobre } \\
\text { mientras jugaba PG? }\end{array}$ & Frecuencia & Porcentaje \\
\hline Muy satisfactorio & 3 & $7 \%$ \\
Satisfactorio & 16 & $36 \%$ \\
Ni satisfactorio Ni deficiente & 19 & $42 \%$ \\
Deficiente & 5 & $11 \%$ \\
Muy deficiente & 2 & $4 \%$ \\
\hline
\end{tabular}

\section{CONCLUSIONES}

La realidad aumentada es una tecnología en auge a nivel mundial (Serino et al., 2016), que hace parte de muchos procesos innovadores y marca una tendencia en la comunicación digital entre jóvenes usuarios Althoff et al. (2016). Como un ejemplo del uso de esta tecnología se encuentra el juego Pokemon Go, el cual involucra dinámicas altamente innovadoras (Howe et al., 2016), acompañadas de una narrativa retro, que son 
responsables de su gran éxito. Sin embargo, desde el punto de vista de los usuarios que hicieron parte del estudio, y de acuerdo con otras investigaciones como la de Wong, (2016), se convierte en rutinario por falta de dinámicas en las actividades colaborativas que podrían generar mayor competitividad e identificar nuevas experiencias en comunidad.

De acuerdo con los resultados de las preguntas cualitativas, es posible establecer que los individuos del estudio muestran un manejo básico de los conceptos relacionados con la realidad aumentada, sin embargo, al profundizar sobre la forma en que funciona y los elementos tecnológicos requeridos para su funcionamiento, sus respuestas no son muy completas. En cuanto a la pregunta cuantitativa, se observa que el $43 \%$ de los usuarios perciben un aprendizaje entre satisfactorio y muy satisfactorio, sobre RA por medio del uso del juego pese a que este no fuera el objetivo del mismo. De acuerdo con esto, se puede decir que un poco menos de la mitad (43\%) de los usuarios del juego Pokémon Go, perciben haber aprendido elementos básicos sobre la realidad aumentada por el solo hecho de jugar y aunque dicho aprendizaje es básico, se puede considerar como un efecto importante en la divulgación de la RA.

Cuando se le preguntó al grupo objetivo sobre nuevas posibles aplicaciones con la RA, la mayoría consideraron aplicaciones de tipo pedagógico, lo cual es una muestra del alto potencial y aceptación que tiene esta tecnología en el sector educativo. De acuerdo con los datos obtenidos, sería conveniente desarrollar juegos educativos que sean basados en la tecnología de la Realidad Aumentada de modo que se obtengan las ventajas de la aplicación georeferenciada, la combinación del mundo real con elementos virtuales y la diversión de los juegos, con un objetivo pedagógico que haga más fácil y divertido el aprendizaje.

Finalmente, aunque Wu et al. (2013) consideran que la RA promueve la curiosidad y motivación en los estudiantes, como resultado de este estudio se puede considerar que las experiencias educativas basadas en la RA pueden enriquecerse con narrativas o elementos de gamificación, que permitan a los estudiantes mantener la nombrada curiosidad y motivación durante más tiempo, de modo que no sean solo juegos basados en RA (como Pokemon Go) o aplicaciones educativas basadas en RA, sino que tomen lo mejor de las dos y se conviertan en juegos de RA con un fin educativo.

\section{REFERENCIAS}

Althoff, T., White, R. W., y Horvitz, E., Influence of Pokémon Go on Physical Activity: Study and Implications, doi: 10.2196/jmir.6759. Journal of Medical Internet Research, 18(12), e315 (2016)

Andújar, J. M., Mejías, A. y Márquez, M. A., Augmented reality for the improvement of remote laboratories: an augmented remote laboratory. IEEE Transactions on Education, 54(3), 492-500 (2011)

Araya, J.J.H., Pokémon Go, un fenómeno inesperado. Investiga. TEC, (28), 4 (2017)

Área, M. y Guarro, A., La alfabetización informacional y digital: Fundamentos pedagógicos para la enseñanza y el aprendizaje competente. Revista Española de Documentación Científica, Monográfico, 46-74 (2012)

Azuma, R.T., A survey of augmented reality. Presence: Teleoperators and virtual environments, 6(4), 355$385(1997)$

Banco Mundial. Informe sobre el desarrollo mundial 2016: Dividendos digitales, cuadernillo del "Panorama general", Washington D.C. Licencia: Creative Commons de Reconocimiento CC BY 3.0 IGO. (2016)

Barry, A., Thomas, G., Debenham, P. y Trout, J., Augmented reality in a public space: The natural history museum, London. Computer, 45(7), $42-47$ (2012)

Beltrán López, G., Geolocalización online: la importancia del dónde, 1ª Ed., Barcelona: UOC. (2016)

Billinghurst, M. y Duenser, A., Augmented reality in the classroom. Computer, 45(7), 56-63 (2012)

Castells, M., La Era de la Información: Economía, Sociedad y Cultura: La sociedad Red, México, Siglo XXI (1999)

Cheng, K. y Tsai, C., Affordances of augmented reality in science learning: Suggestions for future research. Journal of Science Education and Technology, 22(4), 449-462 (2013)

Cuendet, S., Bonnard, Q., Do-Lenh, S. y Dillenbourg, P. Designing augmented reality for the classroom. Computers \& Education, 68, 557-569 (2013) 
De Acevedo, A., Casos y Cosas. Historias verdaderas sobre la realidad de los niños y jóvenes de hoy. Penguin Random House Editorial Colombia (2014)

Díaz, V.M., La emergencia de la Realidad Aumentada en la educación. EDMETIC, 6(1), 1-3 (2016)

Dockterman, D., Great Teaching in the one computer classroom, Tom Snyder Productions, $4^{\mathrm{a}}$ Ed., 35-50 (1997)

Estebanell, M., Ferrés J., Cornell, P. y Codina, D., Realidad Aumentada y códigos QR en Educación en Tendencias Emergentes en Educación con TIC. Asociación Espiral, Educación y Tecnología, 135-155 (2012)

Fabregat, R., Combinando la realidad aumentada con las plataformas de e-elearning adaptativas. https://goo.gl/8bfqBL, ISSN: 1690-7515, Enl@ce Revista Venezolana de Información, Tecnología y Conocimiento, 9 (2), 69-78 (2012)

Guía de Pokémon para padres / tutores. (En línea: https://goo.gl/YnKSo8, acceso: 20 de febrero de 2017) (2015)

Howe, K., B., Suharlim, C., Ueda, P., Howe, D., Kawachi I. y Rimm, E.B. Gotta catch'em all! Pokémon GO and physical activity among young adults: difference in differences study, doi: 10.1136/bmj.i6270, BMJ (2016)

Johnson, L., Adams Becker, S., Cummins, M., Estrada, V., Freeman, A. y Hall, C. NMC Informe Horizon 2016 Edición Superior de Educación. Austin, Texas: The New Media Consortium (2016)

Lee, K., Augmented reality in education and training, doi: 10.1007/s11528-012-0559-3, TechTrends, 56(2), $13-21(2012)$

Marín, V., Posibilidades de uso de la Realidad Aumentada en la educación inclusiva. Estudio de caso. Ensayos, Revista de la Facultad de Educación de Albacete, 31(2), 57-68 (2016)

Milgram, P., y Kishino, F., A taxonomy of mixed reality visual displays. IEICE Transactions on Information and Systems, 77(12), 1321-1329 (1994)

Sanchez, J.M., La e-volución y re-evolución de la tecnología en educación. Relación con los procesos de enseñanza y aprendizaje. $3^{\text {er }}$ Simposio Internacional y $4^{\text {to }}$ Coloquio Regional de Investigación Educativa y Pedagógica (2016)

Serino, M., Cordrey K., McLaughlin L. y Milanaik R.L., Pokémon Go and augmented virtual reality games: a cautionary commentary for parents and pediatricians, doi: 10.1097/MOP.0000000000000409 Current Opinion in Pediatrics. Wolters Kluwer Health, Inc. 28(5), 673-677 (2016)

Strauss, A. L., Corbin, J., y Zimmerman, E., Bases de la investigación cualitativa: técnicas y procedimientos para desarrollar la teoría fundamentada. Universidad de Antioquia, Medellin, Colombia (2002)

Torres, D.R., Realidad Aumentada, educación y museos. Revista ICONO14. Revista científica de Comunicación y Tecnologías Emergentes, 9(2), 212-226 (2011)

Universia, Pokémon go como realidad aumentada puede ser utilizada con fines educativos. (En línea: https://goo.gl/VLzVmY, acceso 20 de febrero de 2017) (2016)

Wenger, E., Comunidades de práctica. Aprendizaje, significado e identidad. Barcelona: Paidós (2001)

Wong J. C. Pokémon No: game's daily active users, downloads and engagement are down. (En línea: https://goo.gl/bKhK1z, acceso 20 de febrero de 2017), The Guardian (2016)

Wu, H., Lee, S. W., Chang, H. y Liang, J. Current status, opportunities and challenges of augmented reality in education. Computers and Education, 62, 41-49 (2013) 